\title{
Ca II H High-Resolution Spectral Monitoring of Active Late-type Dwarfs
}

\author{
J. E. Beckman ${ }^{1}$, R. J. García López ${ }^{1}$, R. Rebolo ${ }^{1}$, \\ and L. Crivellari ${ }^{1,2}$ \\ ${ }^{1}$ Instituto de Astrofísica de Canarias, E-38200 La Laguna, Tenerife, Spain \\ ${ }^{2}$ Osservatorio Astronomico di Trieste, Via G. B. Tiepolo 11, 34131 \\ Trieste, Italy
}

\begin{abstract}
We have monitored $\mathrm{Ca}$ II $\mathrm{H}$ with a spectral resolution of $8 \times 10^{4}$, in a sample of late-type MS stars over a four-year period. The high resolution enables us to add information on velocity fields to the usual flux monitoring. We detect changes in wavelength of different parts of the Ca II H feature, which can be interpreted as velocity fields in the lower chromosphere, with downflow and upflow of order $0.5 \mathrm{~km} \mathrm{~s}^{-1}$, depending on the star. Flux variations in $\mathrm{Ca}$ II $\mathrm{H}$ emission can be ascribed, via velocity tagging, to long-term change in plage cover rather than short-term modulation by (incompletely sampled) rotation cycles.
\end{abstract}

\section{Observations and objectives}

Over a four year period, from 1984 to 1988 , we used the $1.4 \mathrm{~m}$ CAT plus Coudé Echelle Spectrograph of ESO, La Silla (effective spectral resolution $\lambda / \Delta \lambda \sim 8 \times 10^{4}$ at $3970 \AA$ ) to observe systematically, at least once per year, the $\mathrm{Ca}$ II $\mathrm{H}$ line in a sample of late-type dwarfs (late $\mathrm{G}$ and $\mathrm{K}$ ). The objective: to see how much more we could learn from variability in the resolved profile of an emission core than from flux monitoring alone.

\section{Wavelength and flux calibration}

Given the high S:N ratios (at least 200) we could use 5 sharp photospheric absorption lines to establish a photospheric rest frame for each star (see Crivellari et al., 1987 for further details) to a precision of $\pm 2 \mathrm{~m} \AA$, i.e. $\pm 160 \mathrm{~m} \mathrm{~s}^{-1}$; the basic limit is the formation process of these lines in the photosphere. Chromospheric velocities (wavelength displacements detected in the $\mathrm{Ca}$ II $\mathrm{H}$ feature) are absolute, in sense and in amplitude, with respect to this frame. 
Relative calibration of a $\mathrm{Ca}$ II $\mathrm{H}$ profile with respect to the nearby pseudocontinuum is good to a few percent, being limited by non-uniformity ("tilt") in the focal plane illumination. This is the order of the error in comparing chromospheric emission fluxes of the same star at different epochs. The absolute calibration is estimated good to $\pm 20 \%$ (see Rebolo et al., 1989, for more details). This uncertainty applies to intercomparisons of fluxes between different stars.

\section{Velocity gradients and flux variations}

Parameterizing a chromospheric emission line into a positive-going gaussian representing the emission core, and a superposed negative-going gaussian representing the self-absorption, we can specify: $\lambda_{0}$ the photospheric rest wavelength of $\mathrm{Ca}$ II $\mathrm{H}, \lambda_{\mathrm{em}}$ the wavelength of the emission peak, and $\lambda_{3}$ the wavelength of the selfabsorption dip. We can also specify the bisector locus of the measured emission core. We should emphasize one key result: the linear dependence of $\lambda_{\mathrm{em}}-\lambda_{3}$ on the intensity ratio $I_{2 V} / I_{2 R}$ of the blue and red peaks $\left(H_{2 V}\right.$ and $\left.H_{2 R}\right)$; a single linear relation encompassing all the data for all the stars.

Figure 1 shows how the velocity field differs from star to star, when $\lambda_{\mathrm{em}}-\lambda_{0}$ and $\lambda_{3}-\lambda_{0}$ are shown on different arbitrary height scales above the photosphere. These representative plots show that in general $\left|\lambda_{\mathrm{em}}-\lambda_{0}\right|$ is greater than $\left|\lambda_{3}-\lambda_{0}\right|$, and that in general the sense of motion is steady for a given star. We can summarize the bisector information by stating that, in general, the emission core bisectors show negligible velocity gradients.

When we plot the emission flux, measured in a band of width $0.8 \AA$ centred on $\lambda_{\mathrm{em}}$, against $\lambda_{\mathrm{em}}-\lambda_{0}$, for all the stars, two notable observational trends emerge: (a) There is, in general, an absence of correlation between flux and wavelength shift. (b) For a given star the $\lambda_{\mathrm{em}}-\lambda_{0}$ values are not symmetrically dispersed around zero.

\section{Conclusions}

Measuring the profiles has given us much more information than flux monitoring alone could give, although the sparse and infrequent sampling limits the validity of our inferences. We can summarize the main conclusions as follows:

(i) The flux variability observed cannot be due mainly to rotational modulation (a result of our sparse sampling), as shown by the trends (a) and (b) in the previous paragraph, and also because the $\lambda_{\mathrm{em}}-\lambda_{3}$ values are an order of magnitude greater than would be expected from differential rotation with height during the passage of a plage from centre to limb.

(ii) A given star maintains a velocity field in its lower chromosphere whose sense and gradient remain rather stable over periods of years. These velocities are of order $500 \mathrm{~m} \mathrm{~s}^{-1}$, and the sense sampled by the $\mathrm{Ca}$ II $\mathrm{H}$ emission differs from star to star (although in the majority, as for $\alpha$ Cen $\mathrm{B}$, upfow is sampled).

(iii) The ratio of intensities in the $\mathrm{H}_{2 \mathrm{~V}}$ and $\mathrm{H}_{2 \mathrm{R}}$ emission peaks is linearly proportional to the displacement of its self-absorption trough from the overall emission peak, a relation encompassing all stars at all epochs. 

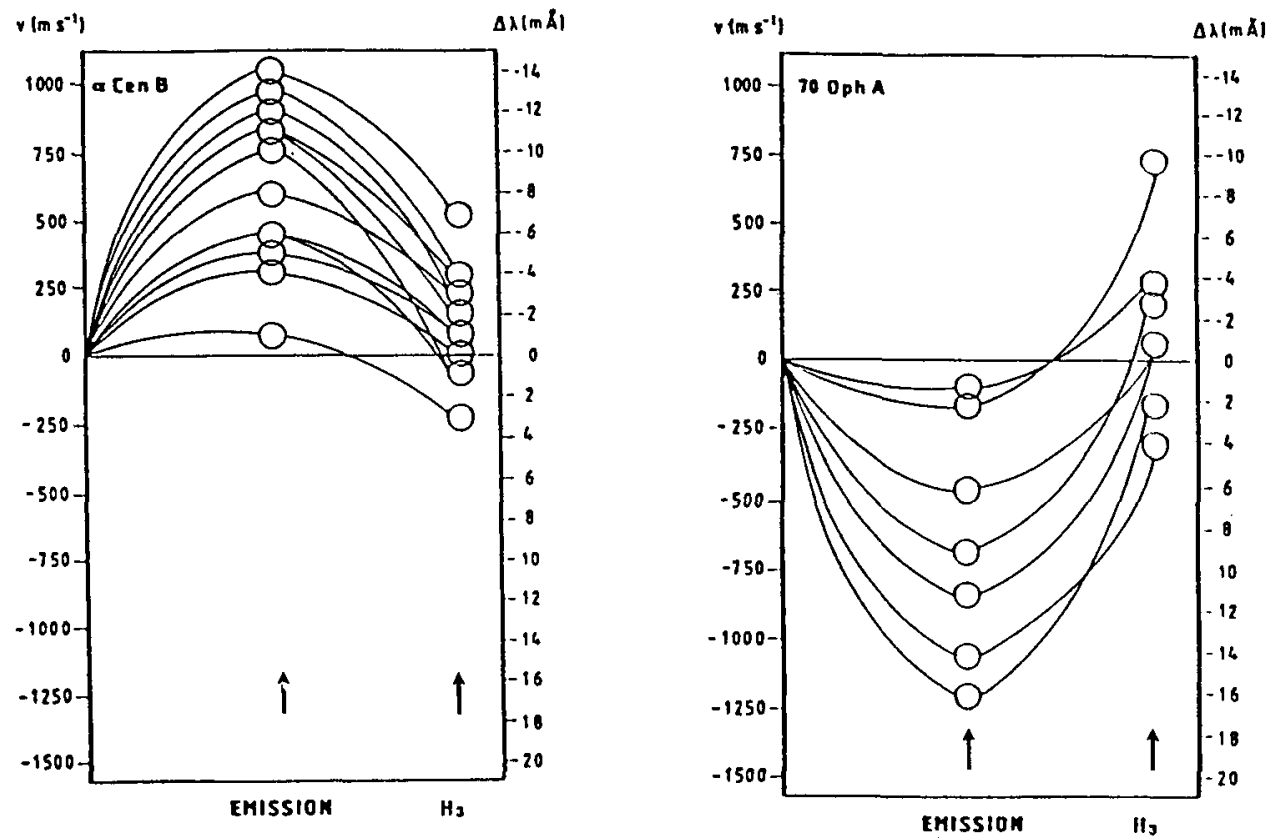

Fig. 1. Shifts $\lambda_{e m}-\lambda_{0}$ of the emission from the photospheric rest wavelength, and $\lambda_{3}-$ $\lambda_{0}$ of the self-absorption from the rest wavelength, against relative height above the photosphere for $\alpha$ Cen B and 70 Oph A.

\section{References}

Crivellari, L., Beckman, J. E., Foing, B. H., Vladilo, G.: 1987, Astron. Astrophys. 174, 127

Rebolo, R., García López, R. J., Beckman, J. E., Vladilo, G., Foing, B. H., Crivellari, L.: 1989, Astron. Astrophys. Suppl. Ser. 80, 135 